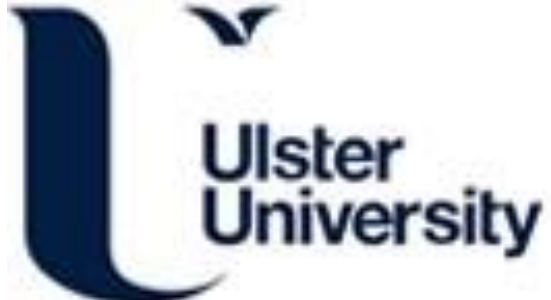

\section{The Power of Augmented Reality and Artificial Intelligence During the Covid-19 Outbreak}

Kerdvibulvec, C., \& Chen, L. (2020). The Power of Augmented Reality and Artificial Intelligence During the Covid-19 Outbreak. In C. Stephanidis, M. Kurosu, H. Degen, \& L. Reinerman-Jones (Eds.), HCl International 2020 - Late Breaking Papers: Multimodality and Intelligence: 22nd HCl International Conference, HCII 2020, Copenhagen, Denmark, July 19-24, 2020, Proceedings (Vol. 12424, pp. 467-476). (Lecture Notes in Computer Science (including subseries Lecture Notes in Artificial Intelligence and Lecture Notes in Bioinformatics); Vol. 12424 LNCS). Springer International Publishing. https://doi.org/10.1007/978-3-030-60117-1_34

Link to publication record in Ulster University Research Portal

Published in:

HCI International 2020 - Late Breaking Papers: Multimodality and Intelligence

Publication Status:

Published (in print/issue): 17/10/2020

DOI:

10.1007/978-3-030-60117-1_34

\section{Document Version}

Author Accepted version

\section{General rights}

Copyright for the publications made accessible via Ulster University's Research Portal is retained by the author(s) and / or other copyright owners and it is a condition of accessing these publications that users recognise and abide by the legal requirements associated with these rights.

\section{Take down policy}

The Research Portal is Ulster University's institutional repository that provides access to Ulster's research outputs. Every effort has been made to ensure that content in the Research Portal does not infringe any person's rights, or applicable UK laws. If you discover content in the Research Portal that you believe breaches copyright or violates any law, please contact pure-support@ulster.ac.uk. 


\title{
The Power of Augmented Reality and Artificial Intelligence During the Covid-19 Outbreak
}

\author{
Chutisant Kerdvibulvech ${ }^{1}$ \\ Liming (Luke) Chen ${ }^{2}$ \\ ${ }^{1}$ Graduate School of Communication Arts and Management Innovation, \\ National Institute of Development Administration, \\ 118 SeriThai Rd., Klong-chan, Bangkapi, Bangkok 10240, Thailand \\ chutisant.ker@nida.ac.th \\ 2 School of Computing, Ulster University \\ Shore Road, Newtownabbey, Co. Antrim, BT37 0QB, United Kingdom \\ 1.chen@ulster.ac.uk
}

\begin{abstract}
The Covid-19 outbreak, the disease elicited by the Severe Acute Respiratory Syndrome Coronavirus-2 (SARS-CoV-2), poses many significant challenges to scientific communities around the world, including computer scientific communities. At the same time, the rise of computer science fueled by advanced in connectivity of social media and smartphones throughout the world, the fields of augmented reality (AR) and artificial intelligence (AI) have recently grown very rapidly. Augmented reality is an emerging field of a physical scene where the things that reside in the physical world are mixed by virtual world, while artificial intelligence is a popular field for the machine simulation of human intelligence that is programmed to see, think and understand like humans. This paper presents the current development of augmented reality and artificial intelligence during the Covid-19 outbreak. First, we highlight a summary of recent tools using augmented reality to tackle the Covid-19 crisis. For instance, augmented reality-based thermal imaging glasses for detecting virus symptoms and methods of augmented reality on educational tasks that help people overcome the isolation for online learning effectively are reviewed. Second, we discuss an overview of recent tools using artificial intelligence to smartly fight against the Covid-19 pandemic. Our discussion include the artificial intelligence methods to approximate and prepare people for prevention the virus, a method for forecasting of the Covid-19 outbreak using non-linear regressive network (NAR) to predict the size, lengths and ending time of the virus, and susceptible-exposed-infectious-removed (SEIR) model for estimating the outbreak trend of the deadly virus. Finally, we suggest benefits and promising future integrations between augmented reality and artificial intelligence to tackle the research problems after the Covid-19 crisis.
\end{abstract}

Keywords: Augmented reality, Artificial intelligence, Data-driven, Covid-19, Coronavirus, Non-linear regressive network, Susceptible-exposed-infectiousremoved 


\section{Introduction}

In recent years, the rise of big data and data-driven economy fueled by advanced in connectivity of social media and smartphones throughout the world, the fields of augmented reality and artificial intelligence have grown very rapidly. Augmented reality is a field of a physical-world environment where the objects that reside in the physical world are mixed by virtual world. Artificial intelligence is a field for the machine simulation of human intelligence that is programmed to see, think and understand like humans. In fact, these two technologies are sometimes distinct, but they can be utilized together to build interactive experiences uniquely. However, ever since the initial report of the Covid-19 outbreak at the capital city of China's Hubei province in the end of year 2019, the virus has affected billions of people from 215 nations and territories with seven million cases and over 400,000 deaths as June 2020 . With the critically evolving outbreak, in this paper, we present the current development of augmented reality and artificial intelligence during the Covid-19 outbreak. Note that 'Co' represents Corona, 'vi' is virus, and 'd' stands for disease, while ' 19 ' represents briefly the year of discovery, which is 2019 , but this paper will generally use the term 'virus' stands for the Covid19 for convenience's sake. The paper is divided into two main sections as follows. To begin with, the first section introduces an overview of recent tools using augmented reality to tackle the virus crisis. Next, the second section explores recent tools using artificial intelligence to fight against the Covid-19 outbreak. Ultimately, we conclude and give a suggestion for future direction.

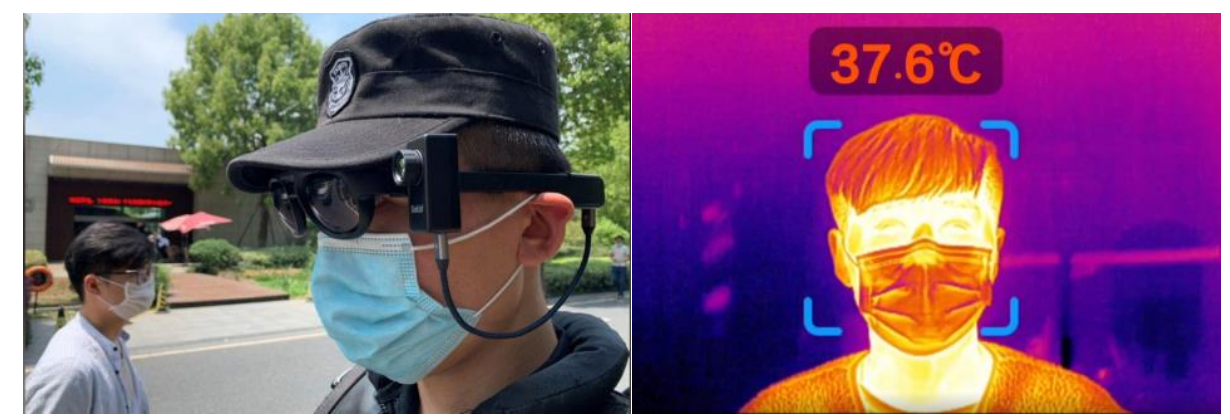

Fig. 1. An example when a security guard wearing the Glass T1 Thermal glasses during the Covid-19 outbreak [1] [2].

\section{$2 \quad$ Augmented Reality to Tackle the Covid-19}

In this section, we discuss a summary of recent tools using augmented reality to tackle the Covid-19 outbreak. For instance, we explore a pair of augmented realitybased thermal imaging glasses, called the Glass T1 Thermal glasses, for detecting 
possibly virus symptoms which is achieved by artificial intelligence start-up called Rokid [1]. The glasses are equipped with a camera and an infrared sensor by integrating thermal infrared sensor into augmented reality and artificial intelligence features, so that it can help people to see others' temperatures on the move in real-time. In fact, the concept of the glasses was started in 2018 and financed by Singapore's Temasek, Switzerland's Credit Suisse and other investors. Then, it has been upgraded into the Glass T1 Thermal glasses for taking multiple temperature readings concurrently in different colors, such as green (non-alert) and orange (alert), for use at the airports, the industrial parks, and the malls to tackle the Covid-19 outbreak in 2020. If the measured temperature is higher than the threshold they set, the readout will be shown on the glasses in an orange color together with a sound for alerting [2]. In the case of sound alerting, we can ask them to go to see the doctor, suggest them to work from home, or give them a facial mask. The Glass T1 Thermal glasses are composed of a Qualcomm CPU, an infrared sensor, 12-megapixel camera with augmented reality features for voice controls and video recordings automatically. In this way, it can identify the temperatures of about 200 people from as far as three meters within several minutes. Figure 1 shows an example when a security guard using the Glass T1 Thermal glasses during the Covid-19 outbreak. Similarly, SenseTime [3] also built a thermal imaging system and installed in railway stations, community centers, and schools in China for tackling the Covid-19 outbreak by spotting people with high temperatures. According to Pratik Jakhar's [4], the SenseTime's system can successfully identify masked faces (i.e., wearing facial masks) and make-up faces with a quick and high accuracy using artificial intelligence's facial recognition technology. Specifically, the system can recognize people in $0.3 \mathrm{sec}-$ onds with $99 \%$ accuracy even if they are wearing glasses and facial masks. This is essential because many countries in Asia, such as China and Thailand, have made the use of facial masks mandatory in some specific places in public such as the malls, the airports, and the railway stations during the Covid-19 outbreak. Therefore, by using a mask algorithm, it can help to flag people who are not wearing a facial mask automatically when they enter some specific buildings for access control. Figure 2 shows the SenseTime's system for recognizing whether people are wearing facial masks in railway stations. A blue color shows people who are wearing facial masks, while a red color displays people who are not wearing facial masks. Also as presented by Maghdid et al. [5], there is a similar research, though they do not exactly use augmented reality technology, for diagnose the virus using artificial intelligence enhanced smartphone with embedded sensors.

In addition, Tely360 built a pair of augmented reality-based smart glasses, called the Ambulance Third Eye module [6], utilized with the Vuzix Blade for telehealth in Thailand during the Covid-19 outbreak to increase patient care productivity and efficiency. The system helps the doctors to communicate what is going on with their patients in the other places immediately. Therefore, it can reduce risks to healthcare personnel staffs to stay near the Covid-19 patients in the Ramathibodi Hospital in Bangkok, Thailand. Note that the number of confirmed active Covid-19 cases in Thailand in the early of June has dipped below 100 because the number of recovering from the disease approximately continues to outstrip the number of new infections recorded almost every day. In fact, many confirmed cases are returnees from overseas. 


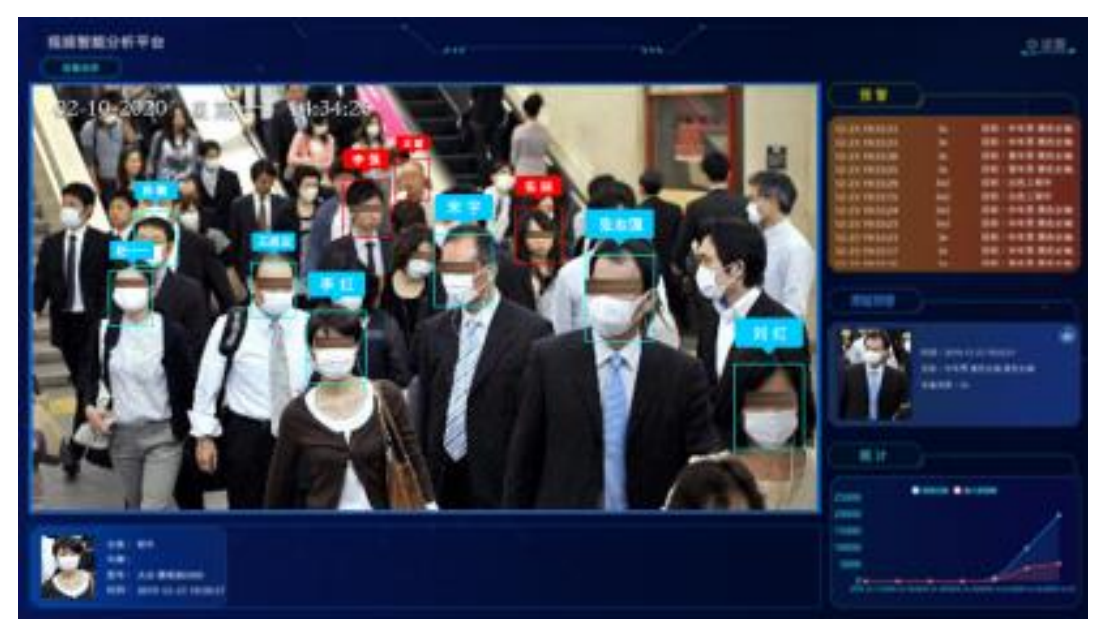

Fig. 2. The SenseTime's system can help to flag people who are not wearing facial masks automatically for building access control using artificial intelligence's facial recognition [3].

Furthermore, since it is essential for schools and universities to have visual communication and interactive experiences rather than over voice during the virus outbreak, we review several methods of augmented reality on educational tasks suggested by Papagiannis [7] that help people overcome the isolation for online learning effectively, such as evaluating the skills of learners remotely and interactively [8]. During the Covid-19 quarantine period, Papagiannis suggests that augmented reality can perform three things well: visualization, annotation and storytelling. First, visualization in augmented reality can allow people to integrate a graphical object into a physical world. A good example of visualization during the Covid-19 crisis is utilizing Microsoft HoloLens and the HoloAnatomy augmented reality software [9] at Case Western Reserve University for helping 185 medical students from different locations in United States. and Canada to learn from homes. As shown in Figure 3, it facilitates medical students to learn and visualize the three-dimensional human anatomy remotely. Second, annotation in augmented reality can guide people and give explanations of everything that's happening around us. A useful example of annotation is Project Tokyo, as proposed recently by Grayson et al. [10] from Microsoft, that aims to help people with disability using augmented reality (HoloLens) and artificial intelligence. The system can help blind people to artificially see by detecting the location of people in the user's environment. Then, it can recognize faces dynamically and relay the essential guidance to them via audio information. Similarly, for using augmented reality to help disabled people is a system of visual and touch communications, as presented in [11]. Third, augmented reality in storytelling can help expressing more unique experiences in both public and private places. A recent example of storytelling in augmented reality is RYOT's work, described in [12] in 2020, by building an augmented reality experience for exploring Oscars dresses from the past fifty years of Academy Awards fashion-the 1970s through the 2010s. In this work, it allows us to put ourselves in the dresses. Therefore, 
by using augmented reality, it can allow people to interact with the iconic Oscars dresses in a new way.

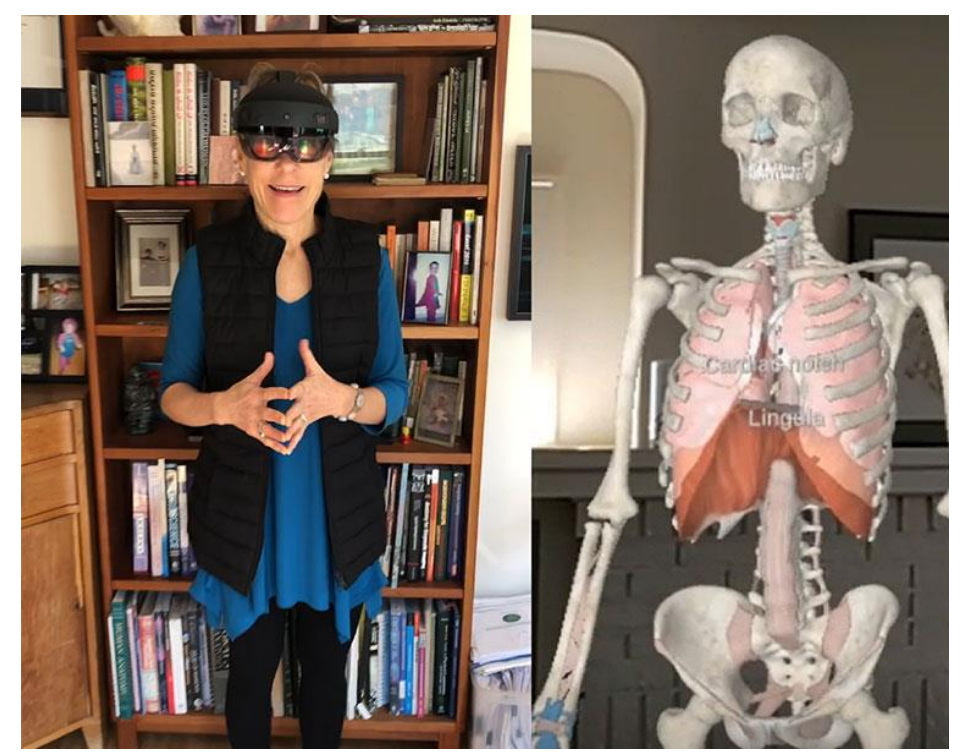

Fig. 3. An anatomy professor from Case Western Reserve University using augmented reality to teach a HoloAnatomy class during the Covid-19 outbreak [9].

\section{Artificial Intelligence to Flight the Covid-19}

This section highlights an overview of recent tools using artificial intelligence to smartly fight against the Covid-19 crisis. For example, a susceptible-exposed-infectious-removed (SEIR) model is utilized in [13], as presented by Yang et al., using population migration data approximately on 23 January 2020 and renewed Covid-19 epidemiological data to estimate the epidemics trend of the virus under public health interventions in China. They also use the past SARS (Severe Acute Respiratory Syndrome) data in 2003 to train the artificial intelligence algorithm for predicting the virus epidemic sizes and peaks. According to their study, they suggest that the implementation of control measures on 23 January 2020 should be essential in reducing the virus epidemic size. The suggestion has later been proved to be true. In addition, an artificial intelligence-based research of Bullock et al. [14] is discussed for using datasets to cope with the virus for three main applications: clinical application, molecular application and societal application. First, according to a clinical application, artificial intelligence, specifically deep learning, can help diagnosis using medical imaging data, such as patterns, computed tomography scans, and electromagnetic radiation images. Then it can track the evolution of disease severity, so that predictions on patient outcomes can be 
achieved using electronic health records. Also, in [15] as reviewed by Shi et al., they explain about the integration of artificial intelligence with computed tomography scans and electromagnetic radiation images, both of which are used by the doctors during this virus crisis for depicting the progress of radiology and medical imaging. Second, according to a molecular application, the virus structure of SARSCoV-2-related proteins can be forecasted by artificial intelligence, and therefore it can possibly help for the discovery and development of medicines and vaccines. Third, according to a societal application, empirical modelling for epidemiological research can be predicted by artificial intelligence. Some examples include predicting the number virus cases given various public policy choices, identifying differences and similarities in the evolution of the pandemic between countries and continents, and verifying the spread and scale of the fake news and hate speech during the virus outbreak. More recently in May 2020, Luengo-Oroz et al. [16] also gives a useful overview of artificial intelligence global partnership to help the response of the virus outbreak for code and model sharing, adapting tools to specific contexts, and partnership between countries.

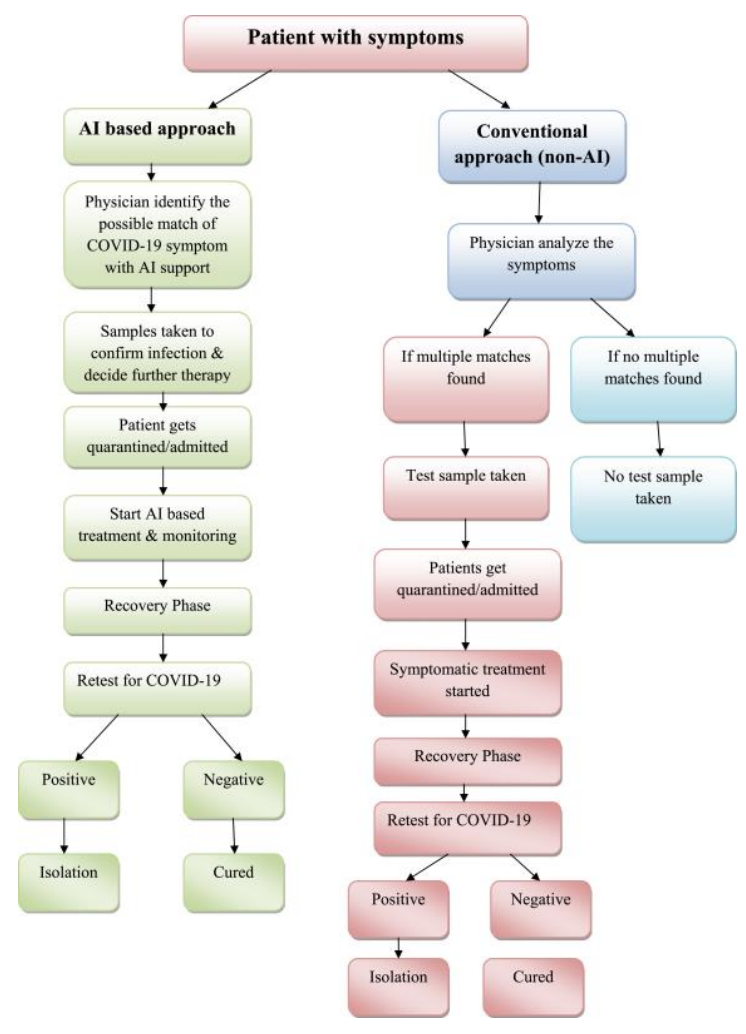

Fig. 4. Overview of artificial intelligence (left in green color) and non-artificial intelligence (right in blue color and red color) based-applications, as presented by Vaishya et al. [17], that support doctors and medical teams to recognize the virus symptoms 
In addition, Vaishya et al. [17] use the artificial intelligence methods to approximate and prepare people for prevention the virus. In fact, it is very crucial and important to slow down the number of new virus cases. This is because one of the reasons why the number of deaths in some countries is so high is that they do not enough medical workers, beds and quarantine facilities to help the patients properly. Figure 4 gives an overview of artificial intelligence and non-artificial intelligence applications that support doctors and medical teams to recognize the virus symptoms, so that they can do both the treatment of the infected patient and the control of virus prevention more effectively. They review seven main applications of artificial intelligence for dealing with the virus outbreak. The seven applications are early diagnosis of the disease, controlling the treatment, testing and tracing of each patient, projection of infected cases and deaths, discovery of medicines and vaccines, decreasing and lightening the load of healthcare people, and prevention of the virus. Furthermore similarly, Naudé [18] gives an early review of six areas where artificial intelligence contributes to deal with the Covid-19 crisis, including quickly warnings, tracing and forecast, strategic data dashboards, diagnosis and prognosis, treatments, and physical control. However, they suggest that artificial intelligence still do not fruitfully impact against the virus outbreak because of both a lack of data and, at the same time, too much data [19]. For this reason, it is suggested that a good balance between information privacy and protection, tools for public health, and precise human-artificial intelligence interaction critically is essential.

Moreover, a real-time method for forecasting of the Covid-19 outbreak using artificial intelligence is presented by Ghazaly et al. [20]. By using non-linear regressive network (NAR), they predict the size, lengths and ending time of this new virus through nine countries, i.e. USA, Brazil, Russian Federation, Spain, Italy, Iran, France, Saudi Arabia, and Egypt. According to the https://www.worldometers.info/coronavirus/ on 6 May 2020, it is important to note that USA, Brazil, Russian Federation, and Spain are the most confirmed coronavirus cases in the world, i.e., 1,902,031 cases, 584,562 cases, 441,108 cases, and 287,406 cases, respectively. In their study, they use the data which is received from the reports of the World Health Organization (WHO), including the virus's infected cases and mortality to analyze and predict the outbreak during the period from 23 March 2020 to 30 July 2020. Figure 5 depicts the map of nine selected countries (above) for prediction and the forecasting results (below) for the cases mean absolute percentage error (MAPE) and the deaths mean absolute percentage error using non-linear regressive network for nine selected countries. The vertical axis represents the cases and the deaths in percentage (error and accuracy). The blue line indicates cases forecast accuracy, while the red line shows deaths forecast accuracy. According to their study for global prediction, training error in the non-linear regressive network is 2.65 percent in cases forecast and 3.22 percent in mortality forecast, respectively. In addition, more recently, Kumar et al. [21] give a useful survey about some modern technologies, focusing on artificial intelligence, machine learning, and data science, that are used to deal with the virus outbreak. One of the examples mentioned is to use artificial intelligence technology for tracking illness of Covid-19 patients with place and time. Nevertheless, they suggest that there are still limitations and constrains for applying the technology for fighting the virus outbreak and pandemic. 


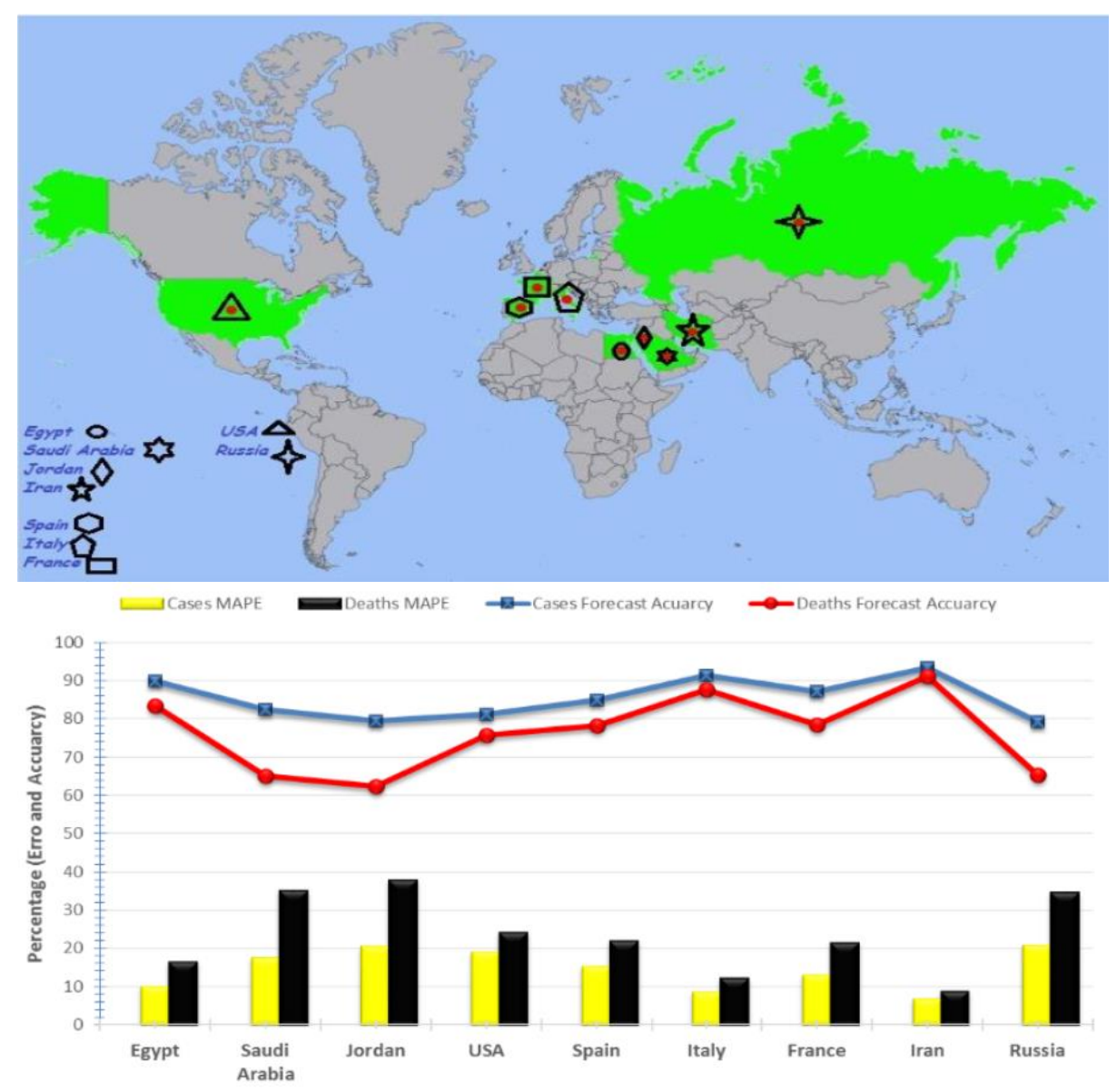

Fig. 5. Forecasting power for the cases mean absolute percentage error and the deaths mean absolute percentage error using non-linear regressive network between 30 March 2020 and 30 July 2020 are proposed by Ghazaly et al. [20].

\section{Conclusion and Suggestion}

This paper has presented the current development of augmented reality and artificial intelligence during the Covid-19 crisis. We first discuss an overview of recent tools using augmented reality to tackle the virus outbreak. After that, we give an overview of recent tools using artificial intelligence technology, including deep learning, to fight against the Covid-19 crisis. We recognize well that dealing with the Covid-19 outbreak is critically difficult worldwide because we must tackle in so many aspects, such as medical aspect, economical aspect, and social aspect. In fact, when the globe is fundamentally interconnected, even though a majority of countries in the world may start to 
successfully contain the virus, we are not truly safe if there are a few countries that still cannot contain it. That is why we suggest applying latest technologies to cope with the outbreak quickly. Augmented reality and artificial intelligence can be good examples to help the virus outbreak in this case. But both technologies require data that are enough, valid, and truthful. Therefore, we believe that the future direction should be cooperation and partnership among countries in term of data exchange management for training artificial intelligence algorithms and building augmented reality-based applications. In other words, when artificial intelligence algorithms are trained with enough data qualitatively and qualitatively, it is possible to create augmented reality-based applications robustly and accurately using computer vision. By applying these technologies, it could help us advance the ways we deal with the virus crisis more smartly, effectively and efficiently.

Acknowledgments. This research presented herein was partially supported by a research grant from the Research Center, NIDA (National Institute of Development Administration).

\section{References}

1. Thomson Reuters, "Chinese startup Rokid sees opportunity with COVID-fighting smart glasses". Retrieved from https://www.reuters.com/article/us-health-coronavirus-china-detection-gl/chinese-startup-rokid-sees-opportunity-with-covidfighting-smart-glasses-idUSKBN22D4TQ on 5 May 2020.

2. Inavate Virtual Events, "Chinese startup develops Covid-19 detecting thermal glasses". Retrieved from https://www.inavateonthenet.net/news/article/chinesestartup-develops-covid-19-detecting-thermal-glasses on 5 May 2020.

3. Jane Li, "China's facial-recognition giant says it can crack masked faces during the coronavirus". Retrieved from https://qz.com/1803737/chinas-facial-recognitiontech-can-crack-masked-faces-amid-coronavirus/ on 10 May 2020.

4. Pratik Jakhar, "Coronavirus: China's tech fights back". BBC World, Retrieved from https://www.bbc.com/news/technology-51717164 on 10 May 2020.

5. H.S. Maghdid, K.Z. Ghafoor, A.S. Sadiq, K. Curran, K. Rabie. "A novel AIenabled framework to diagnose coronavirus COVID 19 using smartphone embedded sensors". Design Study (2020), pp. 1-5.

6. Tely360, "Vuzix Blade Smart Glasses Now Used for COVID-19 Patient Care in Thailand via Tely360's Ambulance 3rd Eye". Retrieved from https://ir.vuzix.com/press-releases/detail/1764 on 11 May 2020.

7. Helen Papagiannis, "3 ways Augmented Reality can have a positive impact on society, COVID-19". World Economic Forum, Retrieved from https://www.weforum.org/agenda/2020/04/augmented-reality-covid-19-positive-use/ on 1 May 2020

8. Chutisant Kerdvibulvech, "Markerless Vision-Based Tracking for Interactive Augmented Reality Game". International Journal of Interactive Worlds, Issue on Serious Games and Interactive Worlds, Vol. 2010, Article ID 751615, 14 pages, 2010.

9. Mike Scott, "HoloAnatomy goes remote, learning goes on during pandemic". Case Western Reserve University, Retrieved from https://thedaily.case.edu/holoanatomy-goes-remote-learning-goes-on-during-pandemic/ on 12 May 2020. 
10. Martin Grayson, Anja Thieme, Rita Marques, Daniela Massiceti, Ed Cutrell, Cecily Morrison “A Dynamic AI System for Extending the Capabilities of Blind People". CHI 2020 Extended Abstracts | April 2020.

11. Chutisant Kerdvibulvech, "A Novel Integrated System of Visual Communication and Touch Technology for People with Disabilities". The 16th International Conference on Computational Science and Its Applications, Lecture Notes in Computer Science, part II, vol. 9787, pp. 509-518, 2016.

12. Nani Sahra Walker, Adam Tschorn, and Marques Harper, "See iconic Oscar dresses and then put yourself in them". Los Angeles Times, February 2020, Retrieved from https://www.latimes.com/lifestyle/story/2020-02-07/oscar-oscarsdresses-celebrities-3d-ar-augmented-reality on 12 May 2020.

13. Zifeng Yang, Zhiqi Zeng, Ke Wang, Sook-San Wong, Wenhua Liang, Mark Zanin, Peng Liu, Xudong Cao, Zhongqiang Gao, Zhitong Mai, Jingyi Liang, Xiaoqing Liu, Shiyue Li, Yimin Li, Feng Ye, Weijie Guan, Yifan Yang, Fei Li, Shengmei Luo, Yuqi Xie, Bin Liu, Zhoulang Wang, Shaobo Zhang, Yaonan Wang, Nanshan Zhong, and Jianxing He, "Modified SEIR and AI prediction of the epidemics trend of COVID-19 in China under public health interventions". Journal of Thoracic Disease, Vol. 12, No. 3, March 2020.

14. Joseph Bullock, Alexandra Luccioni, Katherine Hoffmann Pham, Cynthia Sin Nga Lam, Miguel Luengo-Oroz, "Mapping the Landscape of Artificial Intelligence Applications against COVID-19". 2020.

15. Feng Shi, Jun Wang, Jun Shi, Ziyan Wu, Qian Wang, Zhenyu Tang, Kelei He, Yinghuan Shi, and Dinggang Shen. "Review of Artificial Intelligence Techniques in Imaging Data Acquisition, Segmentation and Diagnosis for COVID-19”. [published online ahead of print, 2020 Apr 16]. IEEE Reviews in Biomedical Engineering, 2020.

16. Miguel Luengo-Oroz, Katherine Hoffmann Pham, Joseph Bullock, Robert Kirkpatrick, Alexandra Luccioni, Sasha Rubel, Cedric Wachholz, Moez Chakchouk, Phillippa Biggs, Tim Nguyen, Tina Purnat, and Bernardo Mariano. "Artificial intelligence cooperation to support the global response to COVID-19”. Nature Machine Intelligence, May 2020.

17. Raju Vaishya, Mohd Javaid, Ibrahim Haleem Khan, and Abid Haleem "Artificial Intelligence (AI) applications for COVID-19 pandemic". Diabetes \& Metabolic Syndrome: Clinical Research \& Reviews, Volume 14, Issue 4, Elsevier, July-August 2020, Pages 337-339.

18. Naudé W. “Artificial Intelligence against COVID-19: An early review”, IZA Discussion Paper no. 13110, Bonn, 2020.

19. Naudé W. "Artificial intelligence vs COVID-19: limitations, constraints and pitfalls”, AI \& Society, 1-5, April 2020, DOI 10.1007/s00146-020-00978-0.

20. Nouby M Ghazaly, Muhammad A Abdel-Fattah, and Abd El-Aziz Ahmed, "Novel Coronavirus Forecasting Model using Nonlinear Autoregressive Artificial Neural Network". Journal of Advanced Science, Volume 29, Number 5, pages 1831-1849, April 2020.

21. Aishwarya Kumar, Puneet Kumar Gupta, and Ankita Srivastava "A review of modern technologies for tackling COVID-19 pandemic". Diabetes \& Metabolic Syndrome: Clinical Research \& Reviews Volume 14, Issue 4, July-August 2020, Pages 569-573. 